\title{
Some New Results on the Lotka-Volterra System with Variable Delay
}

\author{
Yangzi Hu, Fuke Wu, and Chengming Huang \\ School of Mathematics and Statistics, Huazhong University of Science and Technology, Wuhan 430074, China \\ Correspondence should be addressed to Yangzi Hu; huyangzi03@hotmail.com
}

Received 27 January 2014; Accepted 22 July 2014; Published 12 August 2014

Academic Editor: Zhichun Yang

Copyright (C) 2014 Yangzi Hu et al. This is an open access article distributed under the Creative Commons Attribution License, which permits unrestricted use, distribution, and reproduction in any medium, provided the original work is properly cited.

\begin{abstract}
This paper discusses the stochastic Lotka-Volterra system with time-varying delay. The nonexplosion, the boundedness, and the polynomial pathwise growth of the solution are determined once and for all by the same criterion. Moreover, this criterion is constructed by the parameters of the system itself, without any uncertain one. A two-dimensional stochastic delay Lotka-Volterra model is taken as an example to illustrate the effectiveness of our result.
\end{abstract}

\section{Introduction}

Population systems are often subject to environment noise. In our previous papers $[1,2]$, we considered the following stochastic Lotka-Volterra system:

$$
\begin{aligned}
d x(t)=\operatorname{diag}(x(t))\{ & \{[a+A x(t)+B y(t)] d t \\
+ & {[b+D x(t)+E y(t)] d w(t)\} }
\end{aligned}
$$

and its functional form, where $y(t)=x(t-\delta(t))$ with $\delta(t)$ representing variable delay and $\operatorname{diag}(x)=\operatorname{diag}\left(x_{1}, \ldots, x_{n}\right)$ represents the $n \times n$ matrix with all elements zero except those on the diagonal which are $x_{1}, \ldots, x_{n}, a, b \in \mathbb{R}^{n}$ and matrices $A, B, D$, and $E \in \mathbb{R}^{n \times n}$.

Equation (1) may describe dynamics of $n$ species interaction, in which $x_{i}(t)(1 \leq i \leq n)$ represents the population size of $i$ th species depending both on the current states $x(t)$ and on the past state $x(t-\delta(t))$ of all population. From the point of biological view, the following three properties are very important.

(A) The solution of system (1) is positive and nonexplosive; namely, for any positive initial data $\xi$, (1) has a unique positive global solution $x(t, \xi)$.
(B) The solution of system (1) is ultimately moment bounded and time average moment bounded; that is, this global solution $x(t, \xi)$ of (1) satisfies

$$
\begin{gathered}
\limsup _{t \rightarrow \infty} \mathbb{E}|x(t, \xi)| \leq K ; \\
\limsup _{t \rightarrow \infty} \frac{1}{t} \int_{0}^{t} \mathbb{E}|x(t, \xi)|^{2} d s \leq L,
\end{gathered}
$$

where $K$ and $L$ are positive constants independent of $\xi$. These two properties show that, in the sense of average, population size is bounded.

(C) The solution of the system (2) grows at most polynomially; namely, this solution $x(t, \xi)$ of (1) satisfies

$$
\limsup _{t \rightarrow \infty} \frac{\log |x(t, \xi)|}{\log t} \leq 1, \quad \text { a.s. }
$$

There is an extensive literature concerned with these properties of stochastic Lotka-Volterra models. For example, Mao and his coauthors [3-5] discussed the existence and uniqueness of the global positive solution, stochastically ultimate boundedness, and some other asymptotic properties for the stochastic Lotka-Volterra system. References $[6,7]$ discovered that the presence of the environmental noise may 
suppress the potential explosion of the solution in finite time. In our previous work [2], we showed that the environmental noise structure determined whether properties $(\mathrm{A})-(\mathrm{C})$ were affected by the stochastic perturbation parameters or not. In our previous work [1], these three properties were also examined. In this paper, our conclusions will be improved in the following aspects.

(i) In these published works, properties (A)-(C) were given under different conditions, respectively. In this paper, we will give these three properties under the same group of conditions. This is an important improvement since properties (B) and (C) do not imply each other in general.

(ii) In this paper, we will present the conditions, which are easier to be verified, to guarantee properties (A)(C). In these conditions, all parameters are from the models and do not include any uncertain parameters to be determined.

The rest of the paper is arranged as follows. In the next section, we provide some necessary notations and lemmas. Section 3 gives several lemmas to support the main results of this paper. By using Lemmas established in Section 3, Section 4 presents the conditions under which the all desired properties (A)-(C) hold. In Section 5, some simplified cases of model (1) are investigated. Although these models are less general than (1), they have wide applications and satisfy properties (A)-(C) under more simple conditions, which are provided as corollaries of the main theorems. A twodimensional stochastic Lotka-Volterra population model will be examined as an example in Section 6.

\section{Preliminaries}

Throughout this paper, unless otherwise specified, we use the following notations. Let $(\Omega, \mathscr{F}, \mathbb{P})$ be a complete probability space with a filtration $\left\{\mathscr{F}_{t}\right\}_{t \geq 0}$ satisfying the usual conditions; that is, it is right continuous and increasing while $\mathscr{F}_{0}$ contains all $\mathbb{P}$-null sets. $w(t)(t \geq 0)$ is a one-dimensional Brownian motion defined on $\left(\Omega, \mathscr{F}, \mathscr{F}_{t}, \mathbb{P}\right)$.

For any given $x \in \mathbb{R}^{n}$ and $\mathbb{R}^{n}$-valued function $f$, we always assume that

$$
\begin{gathered}
x=\left(x_{1}, x_{2}, \ldots, x_{n}\right)^{\mathrm{T}}, \quad f=\left(f_{1}, f_{2}, \ldots, f_{n}\right)^{\mathrm{T}} ; \\
\operatorname{diag}(x)=\operatorname{diag}\left(x_{i}\right)=\operatorname{diag}\left(x_{1}, x_{2}, \ldots, x_{n}\right) .
\end{gathered}
$$

For matrices $A, B, D$, and $E$ in model (1), we assume that $A=$ $\left[a_{i j}\right], B=\left[b_{i j}\right], D=\left[d_{i j}\right]$, and $E=\left[e_{i j}\right](i, j=1,2, \ldots, n)$. Assume that $A \geq B \Leftrightarrow a_{i j} \geq b_{i j}$ for $i, j=1,2, \ldots, n ; x \gg 0 \Leftrightarrow$ $x_{i}>0$ for $i=1,2, \ldots, n$. Let $\mathbb{R}_{+}=[0, \infty), \mathbb{R}_{+}^{n}=\left(\mathbb{R}_{+}\right)^{n}$, and $\mathbb{R}_{++}^{n}=\left\{x \in \mathbb{R}^{n}: x \gg 0\right\}$. Denote by $|x|$ the Euclidean norm with $x \in \mathbb{R}^{n}$ and $|A|$ is the trace norm of matrix $A$.

Definition 1. Let $A=\left[a_{i j}\right] \in \mathbb{R}^{n \times n}$ satisfy condition

$$
a_{i i}>0 \geq a_{i j} \text { for } i, j=1,2, \ldots, n, i \neq j .
$$

If all eigenvalues of $A$ have positive real parts, $A$ is called an $M$-matrix.
Lemma 2. Suppose that the matrix $A \in \mathbb{R}^{n \times n}$ satisfies condition (6). Then the following conditions are equivalent (see [8]):

(i) $A$ is an M-matrix;

(ii) there exists $c \in \mathbb{R}_{++}^{n}$ such that $A c \gg 0$;

(iii) all of the leading principal minors of $A$ are positive.

For any given symmetric matrix $Q \in \mathbb{R}^{n \times n}$, define

$$
\lambda_{M}^{+}(Q)=\sup _{x \in \mathbb{R}_{+}^{n},|x|=1} x^{\mathrm{T}} Q x
$$

which deduces directly that

$$
\lambda_{M}^{+}(Q) \leq 0 \Longleftrightarrow x^{\mathrm{T}} Q x \leq 0 \quad \text { for any } x \in \mathbb{R}_{+}^{n} .
$$

Let $\delta(t)$ be the variable delay of system (1). Write $\Delta(t)=t-$ $\delta(t)$ with $\delta(t) \in C^{1}\left(\mathbb{R}_{+}, \mathbb{R}_{+}\right)$and $\delta(t) \leq \delta_{0}<\infty$. Then

$$
\eta=: \inf _{t \geq 0} \Delta^{\prime}(t)>0
$$

implies that $\eta \leq 1$ and $\Delta(t)$ is strictly monotone increasing on $[0, \infty)$. Its inverse function $\Delta^{-1}(s)$ is defined on $[-\delta(0), \infty)$, which satisfies

$$
\left(\Delta^{-1}(s)\right)^{\prime}=\frac{1}{\Delta^{\prime}(t)} \leq \eta^{-1}, \quad(s=\Delta(t), t \geq 0) .
$$

Assume that $\tau=\delta(0), C=C\left([-\tau, 0], \mathbb{R}^{n}\right)$, and $C_{++}=$ $C\left([-\tau, 0], \mathbb{R}_{++}^{n}\right) . C$ is a Banach space with the supremum norm. For any given initial data $\xi \in C_{++}, x(t, \xi)$ always represents the solution of (2). When $x(t, \xi) \in \mathbb{R}_{++}^{n}$ for all $t$ in the domain, we call it a positive solution; when $x(t, \xi)$ is defined on $-\tau \leq t<\infty$, it is called a global solution.

Denote that

$$
\begin{array}{cl}
f=a+A x+B y, & g=b+D x+E y, \\
\bar{f}=\operatorname{diag}(x) f, & \bar{g}=\operatorname{diag}(x) g .
\end{array}
$$

Unless otherwise stated, we assume that $x, y \in \mathbb{R}_{++}^{n}$. For any given $V \in C^{2}\left(\mathbb{R}_{++}^{n}\right)$, define

$$
\mathscr{L} V(x, y)=V_{x}(x) \bar{f}(x, y)+\frac{1}{2}\left[\bar{g}^{\mathrm{T}}(x, y) V_{x x}(x) \bar{g}(x, y)\right] .
$$

If $x(t)$ is a positive solution of (1), by the Itô formula and (12), we have that

$$
\begin{aligned}
V(x(t))= & V(x(0))+\int_{0}^{t} L V(x(s)) d s \\
& +\int_{0}^{t} V_{x}(x(s)) \bar{g}(x(s), y(s)) d w(s),
\end{aligned}
$$

where $L V(x(t))=\mathscr{L} V(x(t), y(t))$ with $y(t)=x(t-\delta(t))$.

Let $p$ and $c_{i}(1 \leq i \leq n)$ be positive constants. Define

$$
V(x)=\sum_{i=1}^{n} c_{i} x_{i}^{p}, \quad U(x)=\sum_{i=1}^{n} c_{i}\left(x_{i}^{p}-p \log x_{i}\right) .
$$


Substituting (14) into (12), together with notations in (11), yields that

$$
\begin{gathered}
\mathscr{L} V(x, y)=p \sum_{i=1}^{n} c_{i} x_{i}^{p}\left(f_{i}+\frac{p-1}{2}\left|g_{i}\right|^{2}\right) ; \\
\mathscr{L} U(x, y)=\mathscr{L} V(x, y)+I \\
I=p \sum_{i=1}^{n} c_{i}\left(-f_{i}+\frac{1}{2}\left|g_{i}\right|^{2}\right) .
\end{gathered}
$$

Particularly, when $V=\sum_{i=1}^{n} x_{i}$ and $U=\sum_{i=1}^{n}\left(x_{i}-\log x_{i}\right)$, we have

$$
\begin{aligned}
& \mathscr{L} V(x, y)=x^{\mathrm{T}} f=x^{\mathrm{T}}(a+A x+B y) \\
& \mathscr{L} U(x, y)=\mathscr{L} V(x, y)-\sum_{i=1}^{n} f_{i}+\frac{1}{2}|g|^{2} .
\end{aligned}
$$

For the sake of simplicity, let $\Phi_{\varepsilon}$ represent the following function defined on $\mathbb{R}_{++}^{n} \times \mathbb{R}_{++}^{n}$ :

$$
\Phi_{\varepsilon}=\Phi_{\varepsilon}(x, y)=\sum_{l=1}^{L} a_{l}\left[V_{l}(y)-\eta^{-1} e^{\delta_{0} \varepsilon} V_{l}(x)\right],
$$

where $V_{l} \in C\left(\mathbb{R}_{+}^{n}, \mathbb{R}_{+}\right), \varepsilon$ and $a_{l}(1 \leq l \leq L)$ are nonnegative constants, and $\eta$ is defined in (9). The following lemma plays a key role in this paper (also see $[1,9,10]$ ).

Lemma 3. Let $\Phi_{\varepsilon}$ be given by (18). Suppose that $x(t)=$ $x(t, \xi)\left(\xi \in C_{++},-\tau \leq t<\sigma\right)$ is a positive solution of (1) with $q \leq \varepsilon ;$ then

$$
\int_{0}^{t} e^{q s} \Phi_{\varepsilon}(x(s), y(s)) d s \leq \text { const, } \quad(0 \leq t<\sigma) .
$$

In this paper, const always denotes a positive constant with different values at different places and exact values of these constants are insignificant.

In this paper, we often use the following inequalities:

$$
\begin{gathered}
a^{\alpha} b^{\beta} \leq \frac{\alpha a^{\alpha+\beta}+\beta b^{\alpha+\beta}}{\alpha+\beta} ; \quad(a, b, \alpha, \beta \geq 0, \alpha+\beta>0), \\
(a+b)^{2} \geq \frac{a^{2}}{\rho}-\frac{b^{2}}{\rho-1} ; \quad(a, b \in \mathbb{R}, \rho>1), \\
\left(\sum_{i=1}^{n} c_{i} x_{i}\right)^{2} \leq \sum_{i=1}^{n} c_{i} \sum_{i=1}^{n} c_{i} x_{i}^{2} \quad\left(c_{i} \geq 0, x_{i} \in \mathbb{R}\right) .
\end{gathered}
$$

\section{Main Lemmas}

In order to get the desired properties $(A)-(C)$, we need the following three lemmas. Let us first explain that the notation $o\left(|x|^{\alpha}\right): h(x)=o\left(|x|^{\alpha}\right)$ means that $h(x) \in C\left(\mathbb{R}_{+}^{n}\right)$ with

$$
\lim _{|x| \rightarrow \infty}|x|^{-\alpha} h(x)=0
$$

for $x \in \mathbb{R}_{++}^{n}$.
Lemma 4. Suppose that there exist positive constants $p, \sigma$, $\varepsilon$, $b_{i}$, and $c_{i}(1 \leq i \leq n)$, such that $U=\sum_{i=1}^{n} c_{i}\left(x_{i}^{p}-p \log x_{i}\right)$ satisfies condition

$$
\mathscr{L} U(x, y) \leq \Phi_{\varepsilon}-\sum_{i=1}^{n} b_{i} x_{i}^{\sigma}+o\left(|x|^{\sigma}\right), \quad\left(x, y \in \mathbb{R}_{++}^{n}\right),
$$

where $\Phi_{\varepsilon}$ is defined by (18). Then (1) is positive and nonexplosive; namely, for any given $\xi \in \mathbb{R}_{++}^{n}$, (1) has a unique positive solution $x(t, \xi)$.

Lemma 5. Suppose that there exist positive constants $\varepsilon, b_{i}$, and $c_{i}(1 \leq i \leq n)$, such that $V=\sum_{i=1}^{n} c_{i} x_{i}$ satisfies condition

$$
\mathscr{L} V(x, y) \leq \Phi_{\varepsilon}-\sum_{i=1}^{n} b_{i} x_{i}^{2}+o\left(|x|^{2}\right), \quad\left(x, y \in \mathbb{R}^{n}\right),
$$

where $\Phi_{\varepsilon}$ is given by (18). Then any positive global solution $x(t, \xi)\left(\xi \in C_{++}\right)$of (1) satisfies (2)-(3).

The proofs of the above two lemmas are omitted since two similar approaches can be found in [1].

Lemma 6. Suppose that there exist positive constants $p, \sigma, \varepsilon$, and $b_{i}(1 \leq i \leq n)$, such that the following condition is satisfied:

$$
\begin{aligned}
J & =: \frac{x^{T} f}{e^{T} x}+\frac{p-1}{2}\left(\frac{x^{T} g}{e^{T} x}\right)^{2} \\
& \leq \Phi_{\varepsilon}-\sum_{i=1}^{n} b_{i} x_{i}^{\sigma}+o\left(|x|^{\sigma}\right), \quad\left(x, y \in \mathbb{R}_{++}^{n}\right),
\end{aligned}
$$

where $e=(1,1, \ldots, 1)^{T}, f$ and $g$ are defined by (11), and $\Phi_{\varepsilon}$ is defined by (18). Then any positive global solution $x(t, \xi)(\xi \in$ $\mathrm{C}_{++}$) of (1) satisfies

$$
\limsup _{t \rightarrow \infty} \frac{\log |x(t, \xi)|}{\log t} \leq \frac{1}{p}, \quad \text { a.s. }
$$

Proof. Let $V=\log \left(e^{\mathrm{T}} x\right)\left(x \in \mathbb{R}_{++}^{n}\right)$. Then,

$$
V_{x}=\frac{e^{\mathrm{T}}}{e^{\mathrm{T}} x}, \quad V_{x x}=-\frac{e e^{\mathrm{T}}}{\left(e^{\mathrm{T}} x\right)^{2}} .
$$

By (12) and (26), we have

$$
\mathscr{L} V(x, y)=J-\frac{p}{2}|Z|^{2}, \quad Z=V_{x} \bar{g}=\frac{x^{\mathrm{T}} g}{e^{\mathrm{T}} x} .
$$

Let $h(t)=e^{\varepsilon t} V(x(t))$; then $h(t)=h(0)+I+M(t)$, where

$$
\begin{aligned}
M(t) & =\int_{0}^{t} e^{\varepsilon s} V_{x}(x(s)) \bar{g}(x(s), y(s)) d w(s) \\
& =\int_{0}^{t} e^{\varepsilon s} Z(s) d w(s), \\
I & =\int_{0}^{t} e^{\varepsilon s}[L V(x(s))+\varepsilon V(x(s))] d s \\
& =\int_{0}^{t} e^{\varepsilon s}\left[J-\frac{p}{2}|Z(s)|^{2}+\varepsilon V(x(s))\right] d s .
\end{aligned}
$$


For any given $\theta>1$ and $k \in \mathbb{N}$, by the exponential martingale inequality, we have that

$$
\begin{gathered}
\mathbb{P}\left\{\sup _{0 \leq t \leq k+1}\left[M(t)-\frac{p}{2 e^{\varepsilon(k+1)}} \int_{0}^{t} e^{2 \varepsilon s}|Z(s)|^{2} d s\right]\right. \\
\left.\geq \frac{e^{\varepsilon(k+1)} \log k^{\theta}}{p}\right\} \leq \frac{1}{k^{\theta}} .
\end{gathered}
$$

Since $\sum_{k=1}^{\infty} k^{-\theta}<\infty$, we can employ the Borel-Cantelli lemma to derive that, almost surely, when $k$ is sufficiently large and $k \leq t \leq k+1$, one can get that

$$
\begin{aligned}
M(t) & \leq \frac{e^{\varepsilon(k+1)} \log k^{\theta}}{p}+\frac{p}{2 e^{\varepsilon(k+1)}} \int_{0}^{t} e^{2 \varepsilon s}|Z(s)|^{2} d s \\
& \leq \frac{\theta e^{\varepsilon}}{p} e^{\varepsilon t} \log t+\frac{p}{2} \int_{0}^{t} e^{\varepsilon s}|Z(s)|^{2} d s .
\end{aligned}
$$

Note that $-\sum_{i=1}^{n} b_{i} x_{i}^{\sigma}+o\left(|x|^{\sigma}\right)+\varepsilon V(x) \leq$ const. This, together with (31), (33), and (26), gives that in the sense of almost sure, when $t$ is sufficiently large,

$$
\begin{aligned}
& h(t)-p^{-1} \theta e^{\varepsilon} e^{\varepsilon t} \log t \\
& \leq \text { const }+\int_{0}^{t} e^{\varepsilon s}[J+\varepsilon V(x(s))] d s \\
& \leq \text { const } \\
& \quad+\int_{0}^{t} e^{\varepsilon s}\left[\Phi_{\varepsilon}-\sum_{i=1}^{n} b_{i} x_{i}^{\sigma}(s)+o\left(|x(s)|^{\sigma}\right)+\varepsilon V(x(s))\right] d s \\
& \leq \text { const }+ \text { const } \int_{0}^{t} e^{\varepsilon s} d s \\
& \leq \text { const }\left(1+e^{\varepsilon t}\right)
\end{aligned}
$$

where we have used Lemma 3. This implies that in the sense of almost sure

$$
V(x(t)) \leq p^{-1} \theta e^{\varepsilon} \log t+\text { const }\left(1+e^{-\varepsilon t}\right)
$$

when $t$ is sufficiently large. Therefore,

$$
\limsup _{t \rightarrow \infty} \frac{V(x(t))}{\log t} \leq \frac{\theta e^{\varepsilon}}{p}, \quad \text { a.s. }
$$

Obviously, $\Phi_{\varepsilon}$ is a monotony decrease function of $\varepsilon$, so $\varepsilon$ can be replaced by any $\varepsilon^{\prime} \in(0, \varepsilon)$ in condition (26). Hence we may assume that $\varepsilon$ is sufficiently small. Letting $\theta \rightarrow 1$ and $\varepsilon \rightarrow 0$, we get that

$$
\limsup _{t \rightarrow \infty} \frac{V(x(t))}{\log t} \leq \frac{1}{p}, \quad \text { a.s. }
$$

Note that $V(x) \leq \log |x|$ for $x \in \mathbb{R}_{++}^{n}$. Then (27) follows from (37).

\section{The Main Results}

In this section, let us apply Lemmas 4-6 to establish the main results of this paper. We use the denotations $e=(1,1, \ldots, 1)^{\mathrm{T}}$ and $Q=e e^{T}$.

Theorem 7. Suppose that there exist nonnegative constants $q$, $r, \alpha$, and $\beta$, such that the following conditions are satisfied:

$$
\begin{gathered}
\lambda_{M}^{+}(H) \leq 0, \quad H=\left[\begin{array}{cc}
A+A^{T}+2 q Q & B-r Q \\
B^{T}-r Q & 0
\end{array}\right] ; \\
\lambda_{M}^{+}(F) \leq 0, \quad F=\left[\begin{array}{cc}
D^{T} D-\alpha Q & D^{T} E \\
E^{T} D & E^{T} E-\beta Q
\end{array}\right] ; \\
q>r \eta^{-1} \bigvee \frac{r\left(1+\eta^{-1}\right)+\alpha+\beta \eta^{-1}}{2} .
\end{gathered}
$$

Then for any given $\xi \in C_{++}$, (1) has a unique positive solution $x(t, \xi)$ and this solution satisfies (2)-(4).

Proof. Let us divide this proof into the following three steps.

Step 1. Let $V=\sum_{i=1}^{n} x_{i}\left(x \in \mathbb{R}_{++}^{n}\right)$. Let us test condition (25). By (8) and condition (38), for any given $x, y \in \mathbb{R}_{++}^{n}$ we have that

$$
\begin{aligned}
0 & \geq\left(\begin{array}{ll}
x^{\mathrm{T}} & y^{\mathrm{T}}
\end{array}\right) H\left(\begin{array}{l}
x \\
y
\end{array}\right) \\
& =x^{\mathrm{T}}\left(A+A^{\mathrm{T}}+2 q Q\right)+2 x^{\mathrm{T}}(B-r Q) y \\
& =2 x^{\mathrm{T}}(A x+B y)+2 q\left(e^{\mathrm{T}} x\right)^{2}-2 r\left(e^{\mathrm{T}} x\right)\left(e^{\mathrm{T}} y\right),
\end{aligned}
$$

so

$$
x^{\mathrm{T}}(A x+B y) \leq-q\left(e^{\mathrm{T}} x\right)^{2}+r\left(e^{\mathrm{T}} x\right)\left(e^{\mathrm{T}} y\right) .
$$

By (16) and (42), we get

$$
\begin{aligned}
\mathscr{L} V(x, y) & =x^{\mathrm{T}}(a+A x+B y) \\
& \leq-q\left(e^{\mathrm{T}} x\right)^{2}+r\left(e^{\mathrm{T}} x\right)\left(e^{\mathrm{T}} y\right)+o\left(|x|^{2}\right) \\
& \leq-\left(q-\frac{r}{2}\right)\left(e^{\mathrm{T}} x\right)^{2}+\frac{r}{2}\left(e^{\mathrm{T}} y\right)^{2}+o\left(|x|^{2}\right) \\
& =\Phi_{\varepsilon}-k_{1}\left(e^{\mathrm{T}} x\right)^{2}+o\left(|x|^{2}\right),
\end{aligned}
$$

where

$$
\Phi_{\varepsilon}=\frac{r}{2}\left[\left(e^{\mathrm{T}} y\right)^{2}-\eta^{-1} e^{\delta_{0} \varepsilon}\left(e^{\mathrm{T}} x\right)^{2}\right]
$$

is a function in the form of (18) with $\varepsilon>0$ sufficiently small and

$$
k_{1}=q-\frac{r}{2}-\frac{r}{2} \eta^{-1} e^{\delta_{0} \varepsilon}
$$

By condition (40),

$$
\left.k_{1}\right|_{\varepsilon=0}=q-\frac{r}{2}\left(1+\eta^{-1}\right)>0 .
$$


Since $\varepsilon$ is sufficiently small, we may assume that $k_{1}>0$. Obviously, $\left(e^{\mathrm{T}} x\right)^{2} \geq \sum_{i=1}^{n} x_{i}^{2}\left(x \in \mathbb{R}_{++}^{n}\right)$, so (45) implies (25).

Now, we can apply Lemma 5 to obtain that any global positive solution $x(t, \xi) \quad\left(\xi \in C_{++}\right)$of (1) satisfies (2)-(3).

Step 2. Let $U=\sum_{i=1}^{n}\left(x_{i}-\log x_{i}\right)\left(x \in \mathbb{R}_{++}^{n}\right)$. In this step, we will test condition (24). For any given $x, y \in \mathbb{R}_{++}^{n}$, using condition (39) yields

$$
\begin{aligned}
0 & \geq\left(\begin{array}{ll}
x^{\mathrm{T}} & y^{\mathrm{T}}
\end{array}\right) F\left(\begin{array}{l}
x \\
y
\end{array}\right) \\
& =x^{\mathrm{T}}\left(D^{\mathrm{T}} D-\alpha Q\right) x+2 x^{\mathrm{T}} D^{\mathrm{T}} E y+y^{\mathrm{T}}\left(E^{\mathrm{T}} E-\beta Q\right) y \\
& =|D x|^{2}+2(D x)^{\mathrm{T}} E y+|E y|^{2}-\alpha x^{\mathrm{T}} Q x-\beta y^{\mathrm{T}} Q y \\
& =|D x+E y|^{2}-\alpha\left(e^{\mathrm{T}} x\right)^{2}-\beta\left(e^{\mathrm{T}} y\right)^{2},
\end{aligned}
$$

which implies

$$
|D x+E y|^{2} \leq \alpha\left(e^{\mathrm{T}} x\right)^{2}+\beta\left(e^{\mathrm{T}} y\right)^{2} .
$$

By (17), (44), and (50),

$$
\begin{aligned}
& \mathscr{L} U(x, y) \\
& \leq-\left(q-\frac{r}{2}\right)\left(e^{\mathrm{T}} x\right)^{2}+\frac{r}{2}\left(e^{\mathrm{T}} y\right)^{2}+o\left(|x|^{2}\right) \\
& -\sum_{i=1}^{n} f_{i}+\frac{1}{2}|g|^{2} \\
& \leq-\left(q-\frac{r}{2}\right)\left(e^{\mathrm{T}} x\right)^{2}+\frac{r}{2}\left(e^{\mathrm{T}} y\right)^{2}+o\left(|x|^{2}\right) \\
& + \text { const }|a+A x+B y|+\frac{1}{2}|b+D x+E y|^{2} \\
& \leq-\left(q-\frac{r}{2}\right)\left(e^{\mathrm{T}} x\right)^{2}+\frac{r}{2}\left(e^{\mathrm{T}} y\right)^{2}+o\left(|x|^{2}\right) \\
& + \text { const }|a+A x+B y|+\frac{1}{2}|b|^{2}+b^{\mathrm{T}}(D x+E y) \\
& +\frac{1}{2}|D x+E y|^{2} \\
& \leq-\left(q-\frac{r+\alpha}{2}\right)\left(e^{\mathrm{T}} x\right)^{2}+\frac{r+\beta}{2}\left(e^{\mathrm{T}} y\right)^{2}+o\left(|x|^{2}\right) \\
& +\operatorname{const}(1+|x|+|y|) \\
& =\Phi_{\varepsilon}^{\prime}-k_{2}\left(e^{\mathrm{T}} x\right)^{2}+o\left(|x|^{2}\right)
\end{aligned}
$$

where

$$
\begin{aligned}
\Phi_{\varepsilon}^{\prime}= & \frac{r+\beta}{2}\left[\left(e^{\mathrm{T}} y\right)^{2}-\eta^{-1} e^{\delta_{0} \varepsilon}\left(e^{\mathrm{T}} x\right)^{2}\right] \\
& +\operatorname{const}\left(|y|-\eta^{-1} e^{\delta_{0} \varepsilon}|x|\right)
\end{aligned}
$$

is a function in the form of (18):

$$
k_{2}=q-\frac{r+2}{2}-\frac{r+\beta}{2} \eta^{-1} e^{\delta_{0} \varepsilon} .
$$

Condition (40) implies that $\left.k_{2}\right|_{\varepsilon=0}>0$. Since $\varepsilon>0$ can be sufficiently small, we can get $k_{2}>0$. So (51) can imply condition (24) (choose $\sigma=2$ ). Now we can employ Lemma 4 to obtain that, for any given $\xi \in C_{++}$, (1) has a unique positive global solution $x(t, \xi)$.

Step 3. Choose $p=1$. By (26) we have $J=x^{\mathrm{T}} f / e^{\mathrm{T}} x$. Now we test condition (26). Note that $x^{\mathrm{T}} f=\mathscr{L} V(x, y)$, so by (43) we have

$$
\begin{aligned}
J & \leq-q e^{\mathrm{T}} x+r e^{\mathrm{T}} y+o(|x|) \\
& =\Phi_{\varepsilon}^{\prime \prime}-k_{3} e^{\mathrm{T}} x+o(|x|),
\end{aligned}
$$

where

$$
\Phi_{\varepsilon}^{\prime \prime}=r\left(e^{\mathrm{T}} y-\eta^{-1} e^{\delta_{0} \varepsilon} e^{\mathrm{T}} x\right)
$$

is a function in the form of (18),

$$
k_{3}=q-r \eta^{-1} e^{\delta_{0} \varepsilon} .
$$

By condition (40) we have $\left.k_{3}\right|_{\varepsilon=0}=q-r \eta^{-1}>0$, so we may assume that $k_{3}>0$. Then (54) shows that condition (26) is satisfied (choose $\sigma=1$ ).

Applying Lemma 6 yields that any positive solution $x(t, \xi)\left(\xi \in C_{++}\right)$of (1) satisfies (4). This completes the proof.

Theorem 8. Suppose that there exist nonnegative constants $q$ and $r$, such that condition (38) and the following condition are satisfied:

$$
\begin{gathered}
r Q \geq \pm E \\
\lambda_{M}^{+}(R) \leq 0, \quad R=2 q Q-D-D^{T} ; \\
2 q>r\left(1+\eta^{-1}\right) .
\end{gathered}
$$

Assume that $D \geq 0$,

$$
G=: \operatorname{diag}\left(\eta d_{i i}^{2}\right)-S \text { is an M-matrix, }
$$

where $S=\left[s_{i j}\right], s_{i j}=\bar{e}_{i \bullet} \bar{e}_{i j}, \bar{e}_{i j}=\left|e_{i j}\right|$, and $\bar{e}_{i \bullet}=\sum_{j=1}^{n} \bar{e}_{i j}$. Then the conclusion of Theorem 7 holds.

Proof.

Step 1. By Lemma 2, condition (60) can imply that $G^{\mathrm{T}}$ is an $M$-matrix. Thus, there exists $c \in \mathbb{R}_{++}^{n}$ such that $G^{\mathrm{T}} c \gg 0$. Let $U=\sum_{i=1}^{n} c_{i}\left(x_{i}^{p}-p \log x_{i}\right)\left(x \in \mathbb{R}_{++}^{n}\right), \sigma=2+p$, where $p>0$ is sufficiently small. Now we test condition (24). By (15) we have that

$$
\begin{aligned}
& \mathscr{L} U(x, y) \\
& =p \sum_{i=1}^{n} c_{i} x_{i}^{p}\left(f_{i}+\frac{p-1}{2}\left|g_{i}\right|^{2}\right) \\
& \quad+p \sum_{i=1}^{n} c_{i}\left(-f_{i}+\frac{1}{2}\left|g_{i}\right|^{2}\right)
\end{aligned}
$$




$$
\begin{aligned}
\leq & \text { const }|x|^{p}|f|-\frac{p(1-p)}{2} \sum_{i=1}^{n} c_{i} x_{i}^{p}\left|g_{i}\right|^{2}+\text { const }\left(|f|+|g|^{2}\right) \\
\leq & -\frac{p(1-p)}{2} \sum_{i=1}^{n} c_{i} x_{i}^{p}\left|g_{i}\right|^{2}+\operatorname{const}\left(|x|^{p}|y|+|y|+|y|^{2}\right) \\
& +o\left(|x|^{\sigma}\right) \\
\leq & -\frac{p(1-p)}{2} \sum_{i=1}^{n} c_{i} x_{i}^{p}\left|g_{i}\right|^{2}+\operatorname{const}\left(|y|^{p+1}+|y|+|y|^{2}\right) \\
& +o\left(|x|^{\sigma}\right) \\
= & \Phi_{\varepsilon}-\frac{p(1-p)}{2} \sum_{i=1}^{n} c_{i} x_{i}^{p}\left|g_{i}\right|^{2}+o\left(|x|^{\sigma}\right),
\end{aligned}
$$

where

$$
\Phi_{\varepsilon}=\text { const }\left[|y|^{p+1}+|y|+|y|^{2}-\eta^{-1} e^{\delta_{0} \varepsilon}\left(|x|^{p+1}+|x|+|x|^{2}\right)\right]
$$

is a function in the form of (18). Choose $\rho$ sufficiently large; then

$$
\begin{aligned}
\sum_{i=1}^{n} c_{i} x_{i}^{p}\left|g_{i}\right|^{2} & \\
= & \sum_{i=1}^{n} c_{i} x_{i}^{p}\left(b_{i}+\sum_{j=1}^{n} d_{i j} x_{j}+\sum_{j=1}^{n} e_{i j} y_{j}\right)^{2} \\
\geq & \frac{1}{\rho} \sum_{i=1}^{n} c_{i} x_{i}^{p}\left(\sum_{j=1}^{n} d_{i j} x_{j}\right)^{2}-\frac{1}{\rho-1} \sum_{i=1}^{n} c_{i} x_{i}^{p}\left(b_{i}+\sum_{j=1}^{n} e_{i j} y_{j}\right)^{2} \\
\geq & \frac{1}{\rho} \sum_{i=1}^{n} c_{i} d_{i i}^{2} x_{i}^{\sigma}-\frac{1}{\rho-1} \sum_{i=1}^{n} c_{i} x_{i}^{p}\left(\sum_{j=1}^{n} e_{i j} y_{j}\right)^{2} \\
& -\frac{1}{\rho-1} \sum_{i=1}^{n} c_{i} x_{i}^{p}\left(b_{i}^{2}+2 b_{i} \sum_{j=1}^{n} e_{i j} y_{j}\right) \\
\geq & \frac{1}{\rho} \sum_{i=1}^{n} c_{i} d_{i i}^{2} x_{i}^{\sigma}-\frac{1}{\rho-1} \sum_{i, j=1}^{n} c_{i} s_{i j} x_{i}^{p} y_{j}^{2} \\
& -\operatorname{const} \sum_{i, j=1}^{n} d_{i i}^{2} x_{i}^{\sigma}-\frac{1}{\rho-1} \sum_{i, j=1}^{n} c_{i}^{p} s_{i j} \frac{2 y_{j}^{\sigma}+p x_{i}^{\sigma}}{2+p} \\
& -\sum_{i, j=1}^{n} \frac{p x_{i}^{p+1}+y_{j}^{p+1}}{p+1}-o\left(|x|^{\sigma}\right)
\end{aligned}
$$

$$
\begin{aligned}
= & \sum_{i=1}^{n} c_{i}\left[\frac{d_{i i}^{2}}{\rho}-\frac{p s_{i \bullet}}{(\rho-1)(2+p)}\right] x_{i}^{\sigma} \\
& -\frac{2}{(\rho-1)(2+p)} \sum_{i, j=1}^{n} c_{j} s_{j i} y_{i}^{\sigma}-\mathrm{const} \sum_{i=1}^{n} y_{i}^{p+1}-o\left(|x|^{\sigma}\right) \\
= & -\Phi_{\varepsilon}^{\prime}+\sum_{i=1}^{n} k_{i} x_{i}^{\sigma}+o\left(|x|^{\sigma}\right),
\end{aligned}
$$

where we have used inequalities (20)-(22), $s_{i j}=\bar{e}_{i \bullet} \bar{e}_{i j}, s_{i \bullet}=$ $\sum_{j=1}^{n} s_{i j}$; consider

$$
\Phi_{\varepsilon}^{\prime}=\frac{2}{(\rho-1)(2+p)} \sum_{i, j=1}^{n} c_{j} s_{j i}\left(y_{i}^{\sigma}-\eta^{-1} e^{\delta_{0} \varepsilon} x_{i}^{\sigma}\right)
$$

$$
+ \text { const } \sum_{i=1}^{n}\left(y_{i}^{p+1}-\eta^{-1} e^{\delta_{0} \varepsilon} x_{i}^{p+1}\right)
$$

is a function in the form of (18):

$$
k_{i}=c_{i}\left(\frac{d_{i i}^{2}}{\rho}-\frac{p s_{i \bullet}}{(\rho-1)(2+p)}\right)-\frac{2 \eta^{-1} e^{\delta_{0} \varepsilon}}{(\rho-1)(2+p)} \sum_{j=1}^{n} c_{j} s_{j i}
$$

when $\varepsilon \rightarrow 0, p \rightarrow 0$, and $\rho \rightarrow \infty$,

$$
\rho k_{i} \longrightarrow c_{i} d_{i i}^{2}-\eta^{-1} \sum_{j=1}^{n} c_{j} s_{j i}>0
$$

The last inequality is based on the condition $G^{\mathrm{T}} c \gg 0$. Thus we may assume that $\varepsilon$ and $p$ are sufficiently small, while $\rho$ is sufficiently large; then $k_{i}>0(1 \leq i \leq n)$. Substituting (63) into (61) yields that

$$
\mathscr{L} U(x, y) \leq \Phi_{\varepsilon}^{\prime \prime}-\frac{p(1-p)}{2} \sum_{i=1}^{n} k_{i} x_{i}^{\sigma}+o\left(|x|^{\sigma}\right)
$$

where

$$
\Phi_{\varepsilon}^{\prime \prime}=\Phi_{\varepsilon}+\frac{p(1-p)}{2} \Phi_{\varepsilon}^{\prime}
$$

is a function in the form of (18). Clearly, (67) shows that condition (24) is satisfied.

Now, we can use Lemma 4 to obtain that, for any given $\xi \in C_{++}$, (1) has a unique global positive solution $x(t, \xi)$. 
Step 2. Let $V=\sum_{i=1}^{n} x_{i}$. In this step we test condition (25); for that, we only need to show that conditions (38) and (59) hold. The method is similar to the proof of Theorem 7, Step 1 .

Step 3. Taking any $p \in(0,1)$, now we test condition (26). We can replace $J$ by $(2 /(1-p)) J$ :

$$
\begin{aligned}
& \frac{2}{1-p} J=\frac{2}{1-p} \frac{x^{\mathrm{T}} f}{e^{\mathrm{T}} x}-\left(\frac{x^{\mathrm{T}} g}{e^{\mathrm{T}} x}\right)^{2}=: J_{1}+J_{2}, \\
& J_{1}=\frac{2}{1-p} \frac{x^{\mathrm{T}}(a+A x+B y)}{e^{\mathrm{T}} x} \\
& \leq \operatorname{const}(1+|x|+|y|) \\
&=\operatorname{const}|y|+o\left(|x|^{2}\right), \\
& J_{2}=-\left[\frac{x^{\mathrm{T}} b+x^{\mathrm{T}}(D x+E y)}{e^{\mathrm{T}} x}\right]^{2} \\
&=-\frac{\left(x^{\mathrm{T}} b\right)^{2}+2\left(x^{\mathrm{T}} b\right) x^{\mathrm{T}}(D x+E y)}{\left(e^{\mathrm{T}} x\right)^{2}} \\
& \quad-\left(\frac{x^{\mathrm{T}} D x+x^{\mathrm{T}} E y}{e^{\mathrm{T}} x}\right)^{2} \\
& \leq \operatorname{const}|D x+E y|-\left(\frac{x^{\mathrm{T}} D x+x^{\mathrm{T}} E y}{e^{\mathrm{T}} x}\right)^{2} \\
&=J_{4} \cdot
\end{aligned}
$$

Obviously,

$$
J_{3} \leq \text { const }|y|+o\left(|x|^{2}\right)
$$

Letting $\rho$ be sufficiently large, then inequality (21) gives that

$$
J_{4} \leq-\frac{1}{\rho}\left(\frac{x^{\mathrm{T}} D x}{e^{\mathrm{T}} x}\right)^{2}+\frac{1}{\rho-1}\left(\frac{x^{\mathrm{T}} E y}{e^{\mathrm{T}} x}\right)^{2} .
$$

By condition (58) we have

$$
x^{\mathrm{T}} R x=2 q\left(e^{\mathrm{T}} x\right)^{2}-2 x^{\mathrm{T}} D x \leq 0 ;
$$

thus $x^{\mathrm{T}} D x \geq q\left(e^{\mathrm{T}} x\right)^{2}$, which implies

$$
\left(\frac{x^{\mathrm{T}} D x}{e^{\mathrm{T}} x}\right)^{2} \geq q^{2}\left(e^{\mathrm{T}} x\right)^{2}, \quad\left(x \in \mathbb{R}_{++}^{n}\right) .
$$

Condition (57) derives that

$$
r\left(e^{\mathrm{T}} x\right)\left(e^{\mathrm{T}} y\right)=r x^{\mathrm{T}} Q y \geq\left|x^{\mathrm{T}} E \mathrm{y}\right|, \quad\left(x, y \in \mathbb{R}_{++}^{n}\right) ;
$$

hence,

$$
\left(\frac{x^{\mathrm{T}} E y}{e^{\mathrm{T}} x}\right)^{2} \leq r^{2}\left(e^{\mathrm{T}} y\right)^{2} .
$$

So

$$
J_{4} \leq-\frac{q^{2}}{\rho}\left(e^{\mathrm{T}} x\right)^{2}-\frac{r^{2}}{\rho-1}\left(e^{\mathrm{T}} y\right)^{2} .
$$

Combining (69)-(76) yields

$$
\begin{aligned}
\frac{2}{1-p} J & \leq \text { const }|y|-\frac{q^{2}}{\rho}\left(e^{\mathrm{T}} x\right)^{2}+\frac{r^{2}}{\rho-1}\left(e^{\mathrm{T}} y\right)^{2}+o\left(|x|^{2}\right) \\
& =\Phi_{\varepsilon}^{\prime \prime \prime}-k\left(e^{\mathrm{T}} x\right)^{2}+o\left(|x|^{2}\right),
\end{aligned}
$$

where

$$
\begin{aligned}
\Phi_{\varepsilon}^{\prime \prime \prime}= & \operatorname{const}\left(|y|-\eta^{-1} e^{\delta_{0} \varepsilon}|x|\right) \\
& +\frac{r^{2}}{\rho-1}\left[\left(e^{\mathrm{T}} y\right)^{2}-\eta^{-1} e^{-\delta_{0} \varepsilon}\left(e^{\mathrm{T}} x\right)^{2}\right]
\end{aligned}
$$

is a function in the form of (18),

$$
k=\frac{q^{2}}{\rho}-\frac{r^{2}}{\rho-1} \eta^{-1} e^{\delta_{0} \varepsilon} .
$$

When $\varepsilon \rightarrow 0$ and $\rho \rightarrow \infty$,

$$
\rho k \longrightarrow q^{2}-r^{2} \eta^{-1}
$$

By condition (59), we have $q>r\left(1+\eta^{-1}\right) /(2) \geq r / \sqrt{\eta}$; therefore, $q^{2}>r^{2} \eta^{-1}$. Since we may assume that $\varepsilon$ is sufficiently small and $\rho$ is sufficiently large, there must be $k>0$. Thus, condition (77) deduces that condition (26) is satisfied.

Now, we can apply Lemma 6 to obtain that any positive solution $x(t, \xi) \quad\left(\xi \in C_{++}\right)$of (1) satisfies (27). And then we can get that $x(t, \xi)$ satisfies (4) by letting $p \rightarrow 1$. This completes the proof.

Remark 9. Observing and comparing the conditions of Theorems 7 and 8, the condition they have in common is (38), which only involves parameters from the drift coefficient $f$. Condition (39) in Theorem 7 corresponds to conditions (57), (58), and (60) in Theorem 8 which depend on stochastic disturbances of system (1). Both of them can guarantee the existence and uniqueness of the solution. But it seems that the three conditions of Theorem 8 are more precise than condition (39). Hence, we may expect that Theorem 8 can give more accurate results. However, it needs condition $D \geq$ 0 , which is not requested in Theorem 7. So Theorems 7 and 4.2 have their own strengths and weaknesses.

Remark 10. Theorems 7 and 8 give two classes of conditions under which the desired properties $(\mathrm{A})-(\mathrm{C})$ hold. This is an improvement for our previous results $([1,2])$, since we only established these three results in different conditions, respectively. Moreover, conditions of the two theorems are directly dependent on the parameters of system, except $q$ and $r$. This implies that these conditions are easier to be verified. 


\section{Some Corollaries}

In (1), letting $E=0, D=E=0$, and $B=E=0$, one can get the following "defective" LV systems:

$$
\begin{aligned}
& d x(t)=\operatorname{diag}(x(t))\{[a+A x(t)+B y(t)] d t \\
& +[b+D x(t)] d w(t)\} ; \\
& d x(t)=\operatorname{diag}(x(t))\{[a+A x(t)+B y(t)] d t+b d w(t)\} ;
\end{aligned}
$$

where (83) is equivalent to taking $\delta(t) \equiv 0$ in (1). For (81)(83), we can simplify the conditions of Theorems 7-8 and then obtain corollaries as follows.

Corollary 11. Suppose that there exist nonnegative constants $q$, $r$, and $\alpha$, such that condition (38) and the following conditions are satisfied:

$$
\begin{gathered}
\lambda_{M}^{+}\left(D^{T} D-\alpha Q\right) \leq 0 ; \\
q>r \eta^{-1} \bigvee \frac{r\left(1+\eta^{-1}\right)+\alpha}{2} .
\end{gathered}
$$

Then for any given $\xi \in C_{++}$, (81) has a unique global positive solution $x(t, \xi)$, which satisfies (2)-(4).

Taking $\beta=0$ in Theorem 7, (84) deduces (39)-(40) directly. The following corollary can be found in $[3,4]$.

Corollary 12. Let $D \geq 0, d_{i i}>0(1 \leq i \leq n)$. Then for (81), the conclusion of Corollary 11 holds.

This corollary can be deduced from Theorem 8 . First, let $r=0$ such that condition (57) is satisfied. Second, when $q>0$ is sufficiently small, conditions (58)-(59) are satisfied.

Clearly, Theorem 8 cannot be applied on system (82), but employing Theorem 7 we have the following.

Corollary 13. Suppose that there exist nonnegative constants $q$ and $r$, such that (38) and the following condition are satisfied:

$$
q>\left(r \eta^{-1}\right) \bigvee \frac{r\left(1+\eta^{-1}\right)}{2} \text {. }
$$

Then for any given $\xi \in C_{++}$, (82) has a unique global positive solution $x(t, \xi)$, which satisfies (2)-(4).

Note that when $D=E=0$, we should take $\alpha=\beta=0$ such that condition (39) is satisfied.

Applying Theorem 7 on (83) yields the following.

Corollary 14. Suppose that there exist nonnegative constants $q, r$, and $\alpha$ such that conditions (84) and the following condition are satisfied:

$$
\lambda_{M}^{+}\left(A+A^{T}+2 q Q\right) \leq 0 .
$$

Then for any given $\xi \in C_{++}$, (83) has a unique global positive solution $x(t, \xi)$, which satisfies (2)-(4).

\section{Examples}

Consider the following 2-dimensional LV system:

$$
\begin{aligned}
\frac{d x_{1}(t)}{x_{1}(t)}= & {\left[-8 x_{1}(t)+x_{2}(t)-y_{1}(t)+y_{2}(t)\right] d t } \\
& +\left[\lambda x_{1}(t)+\lambda x_{2}(t)+\mu y_{1}(t)-\mu y_{2}(t)\right] d w(t) \\
\frac{d x_{2}(t)}{x_{2}(t)}= & {\left[x_{1}(t)-7 x_{2}(t)+y_{1}(t)-y_{2}(t)\right] d t } \\
& +\left[\lambda x_{2}(t)-\mu y_{2}(t)\right] d w(t)
\end{aligned}
$$

where $\lambda$ and $\mu$ are nonnegative constants, $y_{i}(t)=x_{i}(t-\tau)(i=$ $1,2)$, and $\tau>0$. Let

$$
\begin{array}{ll}
A=\left(\begin{array}{cc}
-8 & 1 \\
1 & -7
\end{array}\right), & B=\left(\begin{array}{cc}
-1 & 1 \\
1 & -1
\end{array}\right), \\
C=\lambda\left(\begin{array}{ll}
1 & 1 \\
0 & 1
\end{array}\right), & E=\mu\left(\begin{array}{ll}
1 & -1 \\
0 & -1
\end{array}\right) .
\end{array}
$$

By (88), we can compute

$$
\begin{array}{cc}
A+A^{\mathrm{T}}=\left(\begin{array}{cc}
-16 & 2 \\
2 & -14
\end{array}\right), & D^{\mathrm{T}} D=\lambda^{2}\left(\begin{array}{ll}
1 & 1 \\
1 & 2
\end{array}\right), \\
D^{\mathrm{T}} E=\lambda \mu\left(\begin{array}{ll}
1 & -1 \\
1 & -2
\end{array}\right), & E^{\mathrm{T}} E=\mu^{2}\left(\begin{array}{cc}
1 & -1 \\
-1 & 2
\end{array}\right), \\
D+D^{\mathrm{T}}=\lambda\left(\begin{array}{ll}
2 & 1 \\
1 & 2
\end{array}\right), \quad S=\mu^{2}\left(\begin{array}{ll}
2 & 2 \\
0 & 1
\end{array}\right) .
\end{array}
$$

Then, by (38) and (39) we have

$$
\begin{gathered}
H=\left(\begin{array}{cccc}
2 q-16 & 2 q+2 & -1-r & 1-r \\
2 q+2 & 2 q-14 & 1-r & -1-r \\
-1-r & 1-r & 0 & 0 \\
1-r & -1-r & 0 & 0
\end{array}\right) ; \\
F=\left(\begin{array}{cccc}
\lambda^{2}-\alpha & \lambda^{2}-\alpha & \lambda \mu & -\lambda \mu \\
\lambda^{2}-\alpha & 2 \lambda^{2}-\alpha & \lambda \mu & -2 \lambda \mu \\
\lambda \mu & \lambda \mu & \mu^{2}-\beta & -\mu^{2}-\beta \\
-\lambda \mu & -2 \lambda \mu & -\mu^{2}-\beta & 2 \mu^{2}-\beta
\end{array}\right) ; \\
G=\left(\begin{array}{cc}
\lambda^{2}-2 \mu^{2} & -2 \mu^{2} \\
0 & \lambda^{2}-\mu^{2}
\end{array}\right), \\
R=\left(\begin{array}{cc}
2 q-2 \lambda & 2 q-\lambda \\
2 q-\lambda & 2 q-2 \lambda
\end{array}\right) .
\end{gathered}
$$


$\left(1^{\circ}\right)$ Apply Theorem 7. For any given $x \in \mathbb{R}_{+}^{4}$, we have

$$
\begin{aligned}
x^{\mathrm{T}} H x= & (2 q-16) x_{1}^{2}+2(2 q+2) x_{1} x_{2}-2(1+r) x_{1} x_{3} \\
& +2(1-r) x_{2} x_{4}+(2 q-14) x_{2}^{2}+2(1-r) x_{2} x_{3} \\
& -2(1+r) x_{2} x_{4} \\
\leq & (2 q-16) x_{1}^{2}+(2 q+2)\left(x_{1}^{2}+x_{2}^{2}\right) \\
& +(1-r)^{+}\left(x_{1}^{2}+x_{4}^{2}\right) \\
& +(2 q-14) x_{2}^{2}+(1-r)^{+}\left(x_{2}^{2}+x_{3}^{2}\right) \\
= & x_{1}^{2}\left[4 q-14+(1-r)^{+}\right]+x_{2}^{2}\left[4 q-12+(1-r)^{+}\right] \\
& +(1-r)^{+}\left(x_{3}^{2}+x_{4}^{2}\right) .
\end{aligned}
$$

It can be seen that, taking $r \geq 1$ and $q \leq 3$, we have $x^{\mathrm{T}} H x \leq 0$, and then $\lambda_{M}^{+}(H) \leq 0$. Next,

$$
\begin{aligned}
x^{\mathrm{T}} F x= & \left(\lambda^{2}-\alpha\right) x_{1}^{2}+2\left(\lambda^{2}-\alpha\right) x_{1} x_{2}+2 \lambda \mu x_{1} x_{3}-2 \lambda \mu x_{1} x_{4} \\
& +\left(2 \lambda^{2}-\alpha\right) x_{2}^{2}+2 \lambda \mu x_{2} x_{3}-4 \lambda \mu x_{2} x_{4} \\
& +\left(\mu^{2}-\beta\right) x_{3}^{2}-2\left(\mu^{2}+\beta\right) x_{3} x_{4}+\left(2 \mu^{2}-\beta\right) x_{4}^{2} \\
\leq & \left(\lambda^{2}-\alpha\right) x_{1}^{2}+\left(\lambda^{2}-\alpha\right)^{+}\left(x_{1}^{2}+x_{2}^{2}\right)+\lambda \mu\left(x_{1}^{2}+x_{3}^{2}\right) \\
& +\left(2 \lambda^{2}-\alpha\right) x_{2}^{2}+\lambda \mu\left(x_{2}^{2}+x_{3}^{2}\right)+\left(\mu^{2}-\beta\right) x_{3}^{2} \\
& +\left(2 \mu^{2}-\beta\right) x_{4}^{2} \\
= & x_{1}^{2}\left[\lambda^{2}-\alpha+\left(\lambda^{2}-\alpha\right)^{+}+\lambda \mu\right] \\
& +x_{2}^{2}\left[\left(\lambda^{2}-\alpha\right)^{+}+2 \lambda^{2}-\alpha+\lambda \mu\right] \\
& +x_{3}^{2}\left(2 \lambda \mu+\mu^{2}-\beta\right)+\left(2 \mu^{2}-\beta\right) x_{4}^{2} .
\end{aligned}
$$

Clearly, when $\alpha \geq 2 \lambda^{2}+\lambda \mu$ and $\beta \geq\left(2 \mu^{2}\right) \vee\left(\mu^{2}+2 \lambda \mu\right)$, $\lambda_{M}^{+}(F) \leq 0$. Condition (40) is equivalent to $2 q>2 r+\alpha+\beta$. Combining the above equalities yields

$$
\begin{aligned}
6 & \geq 2 q>2 r+\alpha+\beta \\
& \geq 2+2 \lambda^{2}+\lambda \mu+\left(2 \mu^{2}\right) \vee\left(\mu^{2}+2 \lambda \mu\right) ;
\end{aligned}
$$

namely, $\lambda \geq 0$ and $\mu \geq 0$ satisfy

$$
2 \lambda^{2}+\lambda \mu+\left(2 \mu^{2}\right) \vee\left(\mu^{2}+2 \lambda \mu\right)<4 .
$$

Then we can choose nonnegative constants $q, r, \alpha$, and $\beta$, such that conditions (38)-(40) are satisfied; therefore, Theorem 7

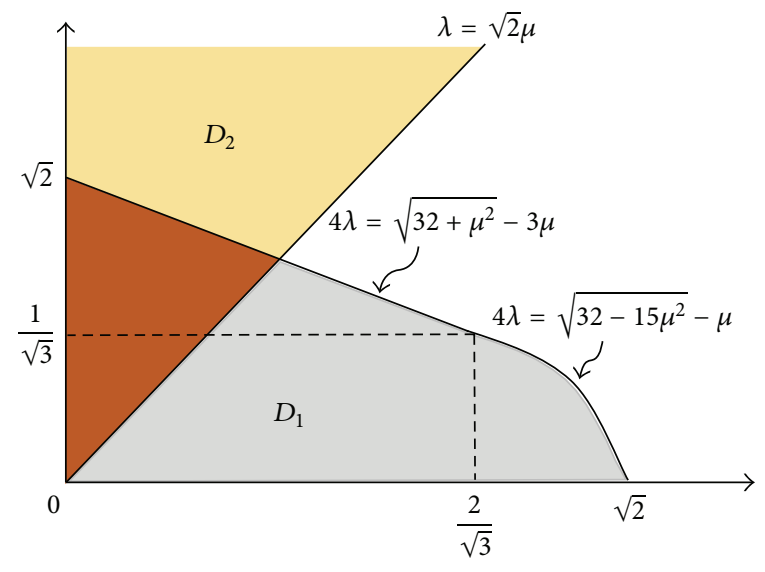

Figure 1: Regions $D_{1}$ and $D_{2}$.

can apply to (87). Through elementary calculation, condition (94) can be expressed as

$$
\lambda< \begin{cases}\frac{\sqrt{32+\mu^{2}}-3 \mu}{4}, & 0 \leq \mu \leq \frac{2}{\sqrt{3}} \\ \frac{\sqrt{32-15 \mu^{2}}-\mu}{4}, & \frac{2}{\sqrt{3}}<\mu<\sqrt{2} .\end{cases}
$$

$\left(2^{\circ}\right)$ Apply Theorem 8 . Obviously $D \geq 0$. By Lemma $2, G$ is an $M$-matrix if and only if $\lambda^{2}>2 \mu^{2}$; that is,

$$
\lambda>\sqrt{2} \mu .
$$

Condition (57) holds $\Leftrightarrow \mu \leq r$, so we may assume $r=\mu$. For any given $x \in \mathbb{R}_{+}^{2}$,

$$
\begin{aligned}
x^{\mathrm{T}} R x & =(2 q-2 \lambda) x_{1}^{2}+2(2 q-\lambda) x_{1} x_{2}+(2 q-2 \lambda) x_{2}^{2} \\
& \leq\left[2 q-2 \lambda+(2 q-\lambda)^{+}\right]\left(x_{1}^{2}+x_{2}^{2}\right) .
\end{aligned}
$$

Obviously, when $q \leq(3 / 4) \lambda, \lambda_{M}^{+}(R) \leq 0$. Let $q=(3 / 4) \lambda$. By condition (96) we have

$$
q=\frac{3}{4} \lambda>\frac{3 \sqrt{2}}{4} \mu \geq \mu=r,
$$

which shows that condition (40) is satisfied. Thus, when condition (96) holds, Theorem 8 can apply to (87). In Figure 1, regions $D_{1}$ and $D_{2}$ are, respectively, decided by conditions (94) and (96) on the $\lambda \mu$ plane. It is easy to see that $D_{1}$ and $D_{2}$ are partially overlapping. Roughly speaking, $D_{2}$ is much larger than $D_{1}$. This means that applying Theorem 8 on model (87) can get more precise results in some sense. This conclusion is consistent with our expectation in Remark 9.

\section{Conflict of Interests}

The authors declare that there is no conflict of interests regarding the publication of this paper. 


\section{Acknowledgments}

The authors would like to thank the referees for their very helpful comments and suggestions. They would also like to thank the National Natural Science Foundation of China (11301198, 11371157, and 91130003) and the Foundation of HUST (2013QN116).

\section{References}

[1] Y. Hu, F. Wu, and C. Huang, "Stochastic Lotka-Volterra models with multiple delays," Journal of Mathematical Analysis and Applications, vol. 375, no. 1, pp. 42-57, 2011.

[2] F. Wu and Y. Xu, "Stochastic Lotka-Volterra population dynamics with infinite delay," SIAM Journal on Applied Mathematics, vol. 70, no. 3, pp. 641-657, 2009.

[3] X. Mao, C. Yuan, and J. Zou, "Stochastic differential delay equations of population dynamics," Journal of Mathematical Analysis and Applications, vol. 304, no. 1, pp. 296-320, 2005.

[4] S. Pang, F. Deng, and X. Mao, "Asymptotic properties of stochastic population dynamics," Dynamics of Continuous, Discrete \& Impulsive Systems A: Mathematical Analysis, vol. 15, no. 5, pp. 603-620, 2008.

[5] A. Bahar and X. Mao, "Stochastic delay population dynamics," International Journal of Pure and Applied Mathematics, vol. 11, no. 4, pp. 377-400, 2004.

[6] A. Bahar and X. Mao, "Stochastic delay Lotka-Volterra model," Journal of Mathematical Analysis and Applications, vol. 292, no. 2, pp. 364-380, 2004.

[7] X. Mao, G. Marion, and E. Renshaw, "Environmental Brownian noise suppresses explosions in population dynamics," Stochastic Processes and their Applications, vol. 97, no. 1, pp. 95-110, 2002.

[8] A. Berman and R. J. Plemmons, Nonnegative Matrices in the Mathematical Sciences, SIAM, Philadelphia, Pa, USA, 1994.

[9] Y. Hu and C. Huang, "Lasalle method and general decay stability of stochastic neural networks with mixed delays," Journal of Applied Mathematics and Computing, vol. 38, no. 1-2, pp. 257278, 2012.

[10] Y. $\mathrm{Hu}$ and C. Huang, "Existence results and the moment estimate for nonlocal stochastic differential equations with time-varying delay," Nonlinear Analysis: Theory, Methods \& Applications, vol. 75, no. 1, pp. 405-416, 2012. 


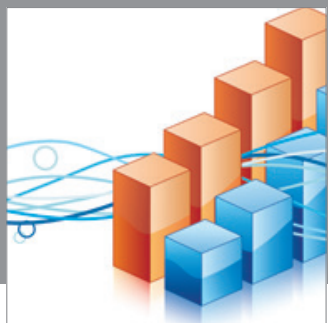

Advances in

Operations Research

mansans

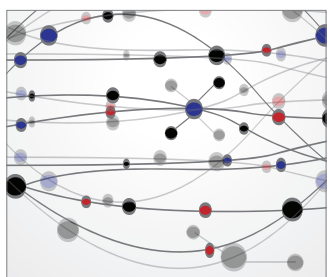

The Scientific World Journal
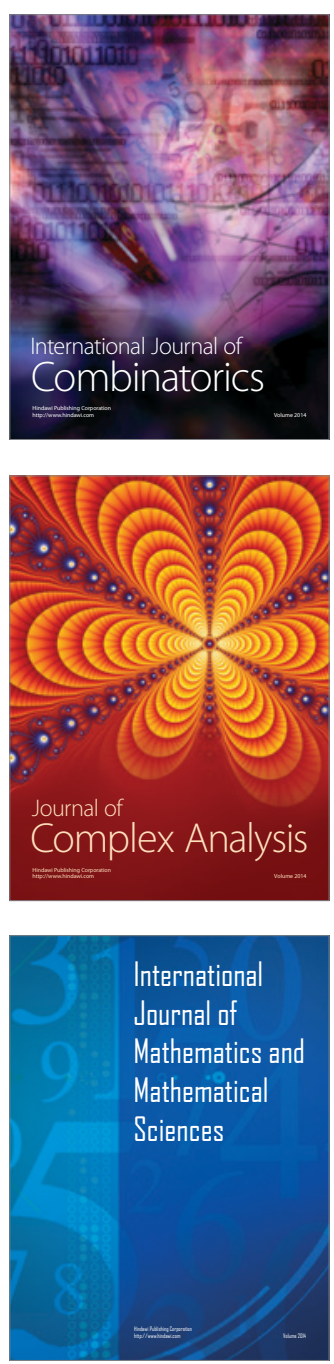
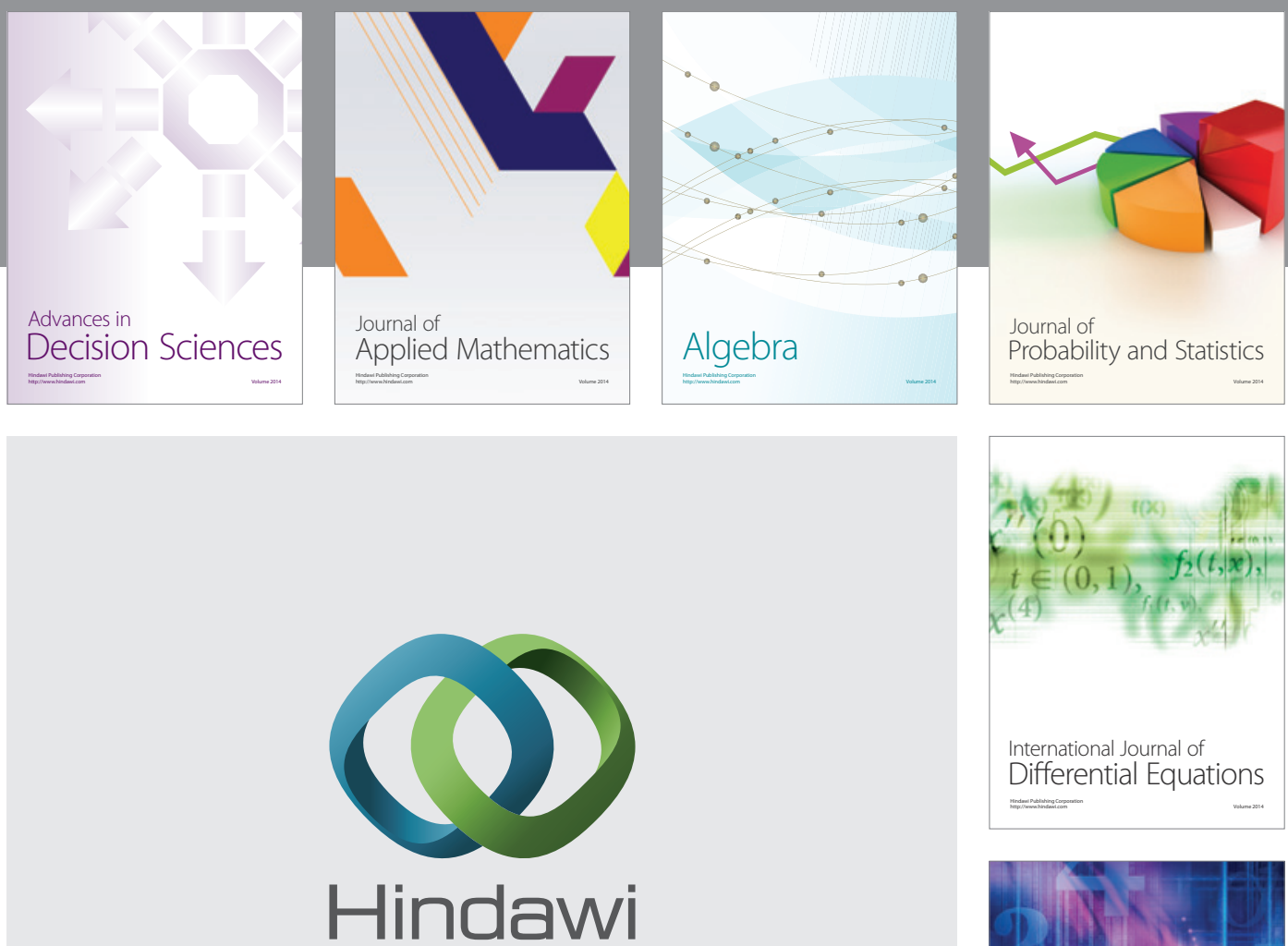

Submit your manuscripts at http://www.hindawi.com
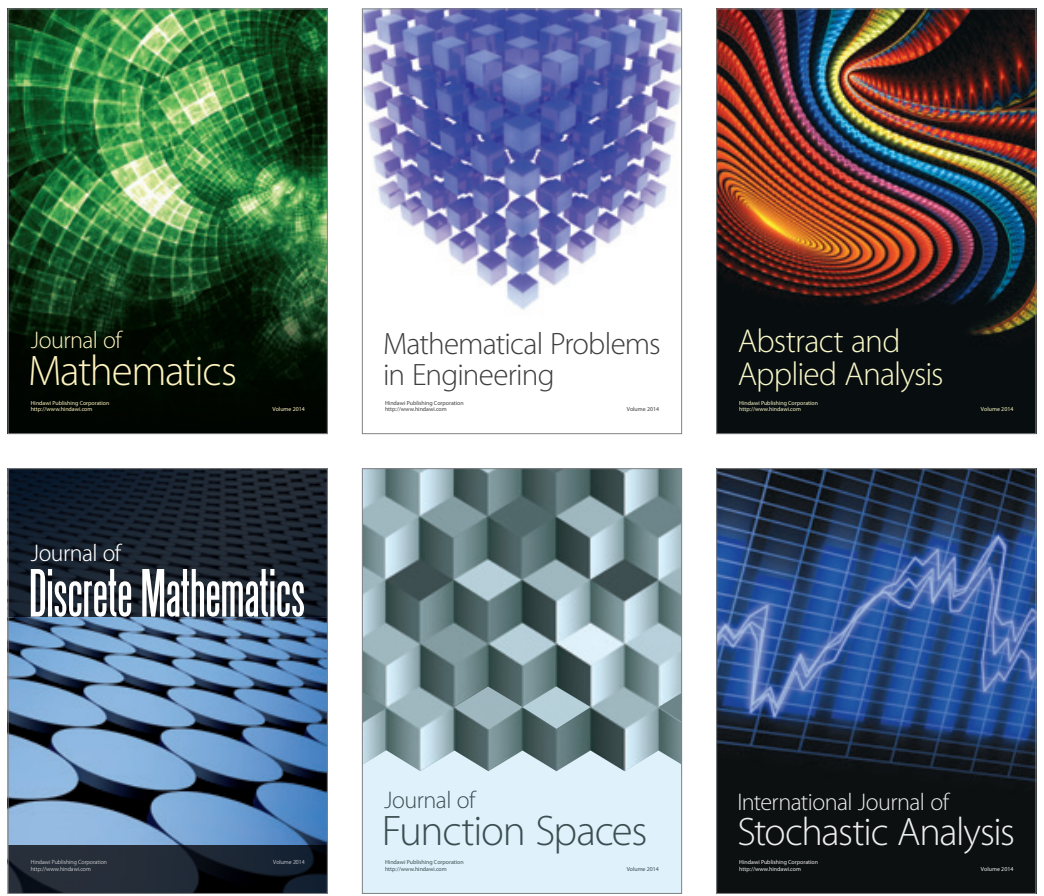

Journal of

Function Spaces

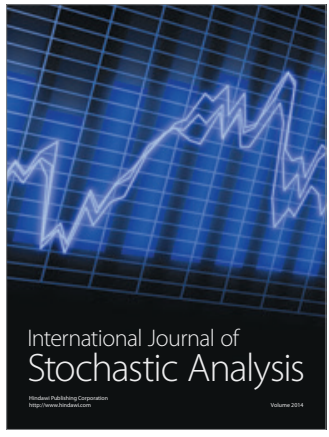

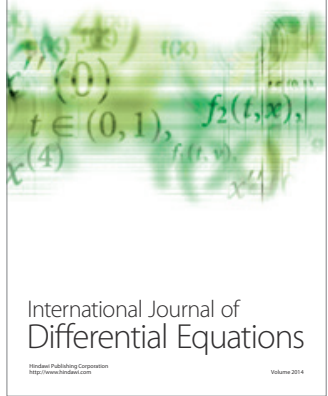
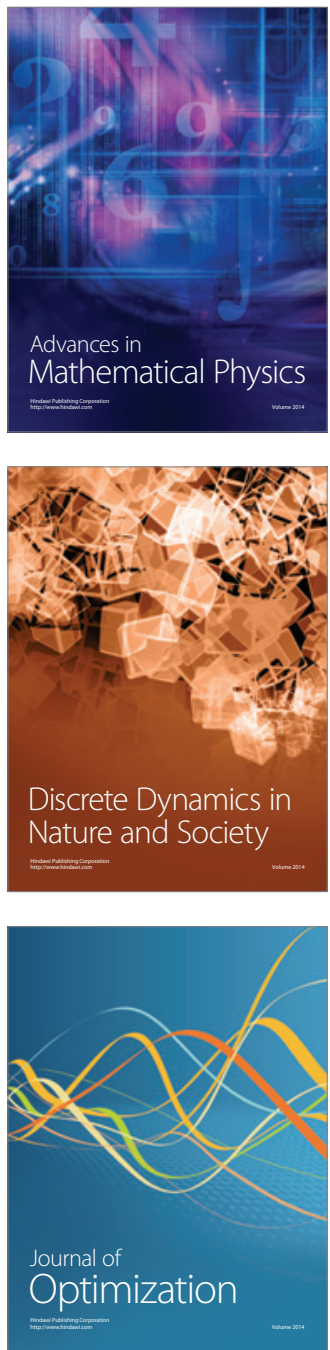